\title{
THE EMOTIONAL AND SOCIAL SKILLS OF STUDENTS IN EGINEERING
}

\author{
Vasile CERNAT ${ }^{\mathbf{1}}$, Liviu MOLDOVAN ${ }^{2}$ \\ 1,2 "PetruMaior” University of TirguMures, Romania \\ Nicolae Iorga Street, no. 1, 540088 TîrguMureş, Romania \\ ${ }^{1}$ cernat.vasile@science.upm.ro \\ 2liviu.moldovan@ing.upm.ro
}

\begin{abstract}
This paper presents some results of the project entitled "Improve your emotional skills" (I$Y E S)$, promoted in the framework of Erasmus + program in a partnership of organizations from 6 European countries. The project concept started from the very poor academic student's performancein engineering and one of the main objectives of the I-YES project is to reduce school failure by increasing student's academic performance. In order to achieve the I-Yes project objective, this paper sought to identify their main emotional and social skill needs and deficits. The research employed both quantitative and qualitative instruments, namely questionnaires and focus-groups. The research suggests that the following variables are most critical in this respect: self-management skills, school interest, self-awareness and well-being at school.
\end{abstract}

Keywords:student's performance, self-management skills, school interest, self-awareness, well-being at school

\section{Introduction}

According to a survey carried out by Broderick [1], social and emotional well-being play a fundamental role. Researcher highlight the fact that success at school come when cognitive skills work in tandem with so-called soft skills like self-control, persistence, social awareness, relationship development and self-awareness [4]. It has been proven that students who develop social and emotional learning skills do better at school [3]. So although the emphasis on academic achievement often captures most of the attention in debates on school reform, important achievements are being made by those who take a more holistic approach to education.

Rethinking Education: Investing in skills for better socio-economic outcomes, (2012) underlines the importance of investing on enhancing students social and emotional skills through which they acquire the knowledge, attitudes and skills to [5, 6]:

- Recognize and manage their emotions;

- Set and achieve positive goals;

- Demonstrate caring and concern for others;

- Establish and maintain positive relationships;

- Make responsible decisions;

- Handle interpersonal situations effectively.

The objective of this paper is to present some results of the project "Improve your emotional skills" (I-YES), promoted in the framework Erasmus+ programme, by Institut pour le Developement et la Formation, Ajaccio, France, in partnership with 5 organizations from Germany, Portugal, Greece Italy and Romania, the "Petru Maior" University of Tirgu Mures [7].

\section{Project Concept}

The I-YES project concept resulted from the applicant and the partner organizations that daily tackle the problems of very poor academic student's performance that often lead to failure at school and to drop outs. Moreover partner organizations work mainly with students form very poor socially and economic backgrounds. Surveys show that adolescents from socially and economically disadvantaged backgrounds are the ones who need supportive intervention as they are more likely to experience failure at school. According to Biegel, et al. [2], research suggests that a significant number of adolescents, especially those form very poor social and economic background, are in need of supportive interventions to promote an emotional well-being and learn adaptive coping skills to reduce negative coping strategies. Many and diverse are the interventions organized according to the national policies and 
individual guidelines. However, all the partners share the following need: provide teachers, trainers and pedagogical staff the necessary resources and pedagogical tools to provide adolescents with a supportive, caring and healthy environment to grow up in and succeed academically.

The I-YES project is innovative in many aspects, and first of all it is based on a student-centered approach. So the first phase is based on the collection of student's data regarding their perspective and needs. The project's tools are based on the data and findings gathered by the online questionnaires and focus groups that involve students and teachers/trainers.

\section{The method of research}

One of the main objectives of the I-YES project is to reduce school failure by increasing student's academic performance through the improvement of classroom behavior and social competence, the increasing of student's attentiveness, student's deeper commitment to school and the increased time devoted to school work. In order to achieve this objective, IYES sought to identify their main emotional and social skill needs and deficits.

For this purpose data was collected from the 6 project partner organizations. The research employed both quantitative and qualitative instruments, namely questionnaires and focus-groups.

The student questionnaire measured three sets of variables: (a) Socio-emotional skills based on Goleman's Four-Cluster Emotional Competency Model; (b) Behavioral problems at school and school atmosphere; (c) Students' perception of the attitudes towards school and learning expressed by relevant social actors. The student questionnaires have been applied to a total of 263 teens.

The teacher questionnaire measured trainers' perception of trainees' socio-emotional skills. The teacher questionnaires have been applied to a total of 155 trainers.

The focus groups measured the same variables but instead of closed ended items, they gathered more elaborate and detailed information and captured issues generated by the respondents. The focus groups collected information from a total of 113 trainees and 38 trainers.

\section{Results}

The main findings for the objective of the research, namely the students' emotional and social skill needs and deficits revealed that the main needs and deficit of the students concern the following four important social and emotional processes:

(1) Self-management skills, especially the selfmanagement of negative emotions (e.g., sadness, anxiety). Students needs concerned (I) the onset of emotions (e.g., students were unhappy that they experience negative emotions in circumstances that should not automatically generate such emotions), (II) the offset of emotions (e.g., students were unhappy by their inability to suppress negative feelings) and(III) their aftermath (students expressed discontentthe disruptive cognitive and behavioral consequences of negative emotions). Moreover, students' responses also made apparent their need for a (IV) better knowledge of what emotions are and how they operate and affect our lives.

(2) School interest. Both students and teachers agreed that this variable represents an important need, but there was a significant discrepancy in how these groups explained the issues. Whereas students tended to attribute the problem to the behavior of the teachers (e.g., lack of teacher involvement in class, uninteresting teaching methods and contents), teachers attributed the problem mainly to negative peer influence.

(3) Self-awareness.Both students and teacher responses indicate that self-awareness skills are represent an essential variable that needs to be improved in order to obtain better results at school. Especially teacher tend to attribute many school related issues to a lack of self-esteem or self-confidence. Students themselves tended to talk less often about such needs and those who did mention low self-awareness tended to also report additional issues, like behavioral problems at school.

(4) Well-being at school.The questionnaire data showed that students' well-being had three main dimensions and school related wellbeing had the lowest average scores, suggesting that students are significantly less content with their experiences at school than with other aspects of their social life. In the same vein, the responses collected via focus groups pointed to a similar issue, which seems closely related to that of school interest.

The mean values of the scales can be seen more intuitively in Figure 1, which depicts the means and their associated confidence intervals. In a strict sense, the difference between two scores is statistically significant (i.e., there is a low probability to observe such data under the null hypothesis) if the confidence intervals do not overlap (i.e., $\mathrm{p}<.01$ ). Overall, the largest values were recorded for parental attitudes, which were close to the maximum of the scale (6.30) whereas serious behavioral issues and negative school atmosphere had the lowest scores (1.91 and 2.52 respectively). If we focus on socio- 
emotional skills we can notice that relationship management and empathy had significantly higher scores than the other variables, whereas self-control, school interest, and self-esteem had lower scores. Finally, differences between the variables pertaining to wellbeing and perception of school attitudes among various social actors were smaller with school related wellbeing tending to score lower than the other variables.

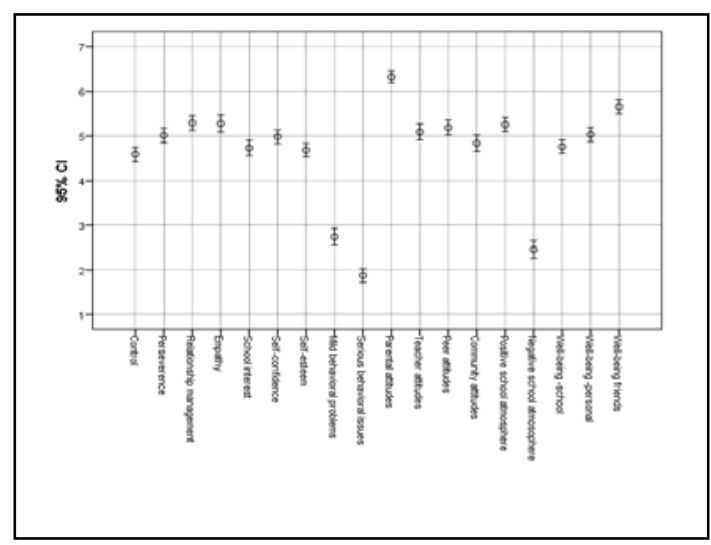

Fig. 1. Means and 95\% confidence intervals of the student's questionnaire's scales

Behind these general results, the analyses also found significant gender and national differences:

(1) Compared to boys, girls tended to report less frequent behavioral and attitudinal problems at school. Nevertheless, the size of these differences was small.

(2) Country had strong effects, especially on behavioral issues and negative school atmosphere. In essence, Greek and Romanian samples tended to report less problems at school than the other national samples, especially the French sample. Although these findings are consistent with the teacher questionnaire results and even with data obtained by educational international tests, in the end we tended to attribute these differences to sampling. After all, the focus group results revealed more similarities than differences.

As revealed in Figure 2, for all measures, whether their differences were significant or not, males scored lower than females on positive attitudes and higher on negative attitudes. It must be stressed that in terms of the magnitude of the effects, gender was less important than country. We used as a measure of effect size $\eta^{2}$ (eta squared).

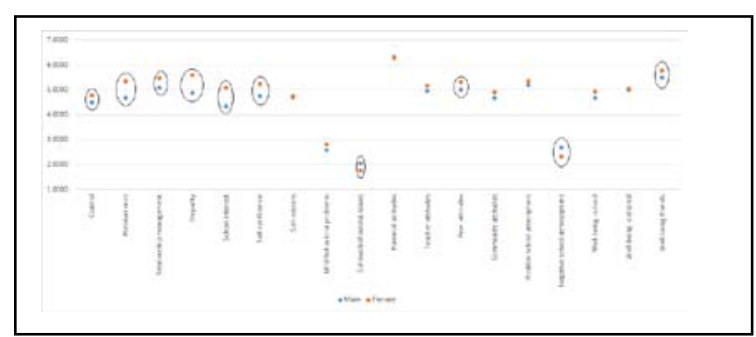

Fig. 2. Means of the student's questionnaire's scales by gender. Circled differences are statistically significant a $\mathrm{p}<.05$

Conventionally, values above .26 indicate strong effects; values lower than .02 indicates small effect whereas values around .13 indicate medium effects. In the case of gender, $\eta^{2}$ ranged between .001 and .062, whereas for country these values ranged between .022 and .255. For gender, the largest differences were observed on perseverance $\left(\eta^{2}=\right.$ $.062)$, school interest $\left(\eta^{2}=.056\right)$, empathy $\left(\eta^{2}=.047\right)$ and self-confidence $\left(\eta^{2}=.026\right)$. For country, the largest differences were observed on serious behavioral problems $\left(\eta^{2}=.255\right)$, mild behavioral problems $\left(\eta^{2}=.224\right)$, negative school atmosphere $\left(\eta^{2}\right.$ $=.177)$ and relationship management $\left(\eta^{2}=.168\right)$. These differences are depicted in Figure 3. It can be seen that another important difference between the gender and country effects is that the first ones concern mostly socio-emotional skills variables whereas the later concern mostly negative behaviors and school atmosphere. Regarding the country effects it seems that behavioral problems seem to be most important in the French sample, whereas participants from the Greek and Romanian samples tend to report the least problematic behaviors and attitudes. In the teacher sample country was a far less important predictor (only the effect on relationship management was significant).

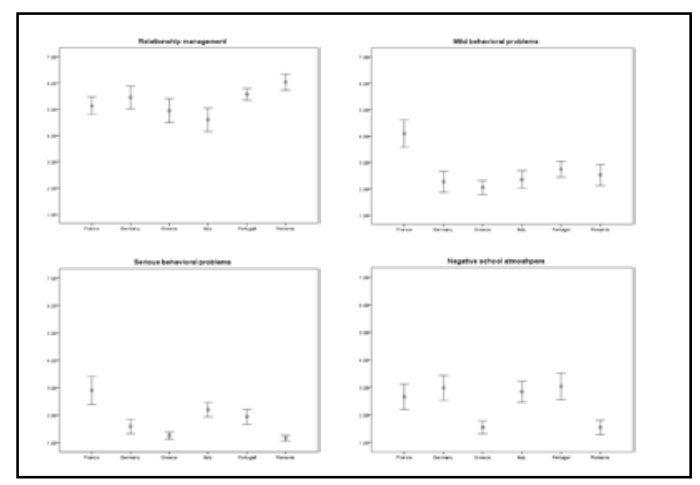

Fig. 3. Means of the variables for which country had the largest effects

Finally, country and gender interacted significantly on several variables and Figure 4 shows the two most important such interactions. These graphs suggest 
that, for negative school atmosphere and selfconfidence, the effects of gender vary as a function of the nationality of respondents.

Specifically, Portugal was the only country in which the general tendency of girls to score higher on positive variables and lower on negative variables was reversed whereas the French sample had a much larger discrepancy between girls and boys on selfconfidence.

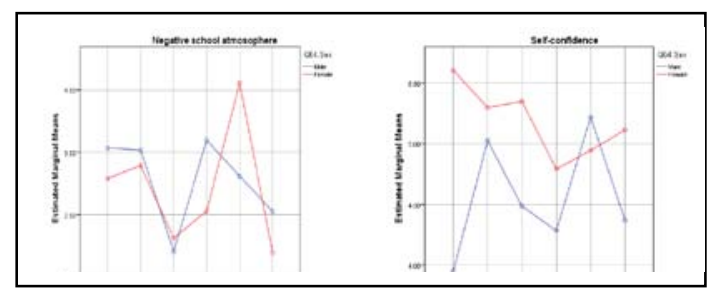

Fig. 4. Interaction of country and gender on perceived negative school climate and self-confidence

When interpreting these data it is important to underline that not only that we worked with convenience samples but the size of the national samples was quite small. Even under ideal sampling conditions, small samples sizes introduce a great amount of noise around the signal and, for this reason, observed effects can be way off their actual values.

Figure 5a shows the results of a simulation conducted in $\mathrm{R}$ in which we extracted 190.000 samples (10.000 per each sample size) from populations with identical means and standard deviations. It can be noticed that for sample sizes similar to the national subsamples from our study the oscillations of the observed effect sizes (Cohen's d) around the true population mean difference are quite high and that these oscillations became gradually shorter and stabilize after samples sizes greater than 200. These simulations results imply that we should be more confident in the results obtained in the general sample and less confident in the comparisons of the national subsamples.

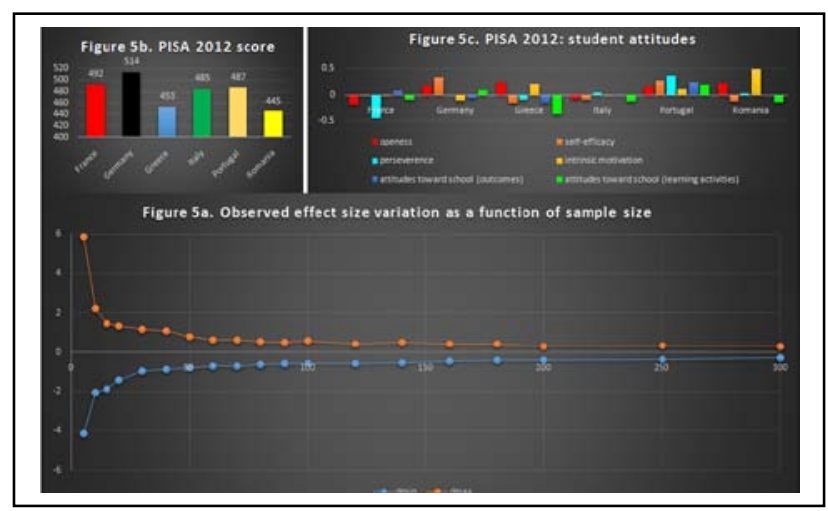

Fig. 5. Means scores among student and teacher sample on the relationship management variables.
From these observations it does not automatically follow that the results obtained in the current samples are inherently flawed. For example, one could think that, since in general teenagers in France get higher scores at international tests than many European counterparts that fact that in our sample French teenagers reported more problematic school related behaviors this would imply that this particular sample does not speak well of the general situation in France. Similarly, Romanian and Greek teenagers generally score lower than their European colleagues in international educational tests, yet in our current sample they report some of the most positive attitudes and behaviors. Obviously, no one would expect a small sample to capture well such issues.

However, as showed in Figure $5 b$ and $5 c$, similar findings are revealed even by international tests that are applied to large and representative national samples. German teenagers had the highest scores at PISA 2012 and Romanian teenagers the lowest, yet Romanian teenagers reported a much stronger intrinsic motivation to learn compared to their German colleagues. Similarly, French teenagers obtained higher scores than Italian, Portuguese, Greek, and Romanian teenagers, yet they report some of the most negative learning attitudes. Moreover, in the teacher sample, the differences in relationship management had the same pattern (i.e., Romania had the highest scores and Italy the lowest).

\section{Conclusion}

The objective of the paper is to identify the main socio-emotional needs of the students in engineering. The research suggests that the following variables are most critical in this respect:

(1) Self-management skills, especially the selfmanagement of negative emotions (e.g., sadness, anxiety). Students needs concerned (I) the onset of emotions (e.g., students were unhappy that they experience negative emotions in circumstances that should not automatically generate such emotions), (II) the offset of emotions (e.g., students were unhappy by their inability to suppress negative feelings) and (III) their aftermath (students expressed discontent the disruptive cognitive and behavioral consequences of negative emotions). Moreover, students' responses also made apparent their need for a (IV) better knowledge of what emotions are and how they operate and affect our lives.

(2) School interest. Both students and teachers agreed that this variable represents an important need, but there was a significant discrepancy in how these groups explained the issues. Whereas students tended to 
attribute the problem to the behavior of the teachers (e.g., lack of teacher involvement in class, uninteresting teaching methods and contents), teachers attributed the problem mainly to negative peer influence.

(3) Self-awareness. Both students and teacher responses indicate that self-awareness skills are represent an essential variable that needs to be improved in order to obtain better results at school. Especially teacher tend to attribute many school related issues to a lack of self-esteem or self-confidence. Students themselves tended to talk less often about such needs and those who did mention low self-awareness tended to also report additional issues, like behavioral problems at school.

(4) Well-being at school. The questionnaire data showed that students' well-being had three main dimensions and school related wellbeing had the lowest average scores, suggesting that students are significantly less content with their experiences at school than with other aspects of their social life. In the same vein, the responses collected via focus groups pointed to a similar issue, which seems closely related to that of school interest.

\section{Acknowledgements}

Supported by the grant no. 2015-1-FR01-KA202015115 financed by the European Commission. This publication reflects the views only of the author, and the Commission cannot be held responsible for any use, which may be made of the information contained therein.

\section{References}

[1] Broderick, P. (2013), Learning to Breathe: A mindfulness curriculum for adolescents. Oakland, CA: New Harbinger.

[2] Biegel, G. M., Brown, K. W., Shapiro, S. L, \& Schubert, C. (2009), Mindfulness-based stress reduction for the treatment of adolescent psychiatric outpatients: A randomized clinical trial. Journal of Clinical and Consulting Psychology, pp. 855-866.

[3] Friedlaender, D., Burns, D., Lewis-Charp, H., Cook-Harvey, C. M., Zheng, X., \& DarlingHammond, L. (2014), Student-centered schools: Closing the opportunity gap. Stanford, CA: Stanford Center for Opportunity Policy in Education. [Online]. Available: https://edpolicy.stanford.edu/sites/default/files/s cope-pub-student-centered-research-brief.pdf.

[4] Thapa, A., Cohen, J., Guffey, S., \& HigginsD'Alessandro, A. (2013). A review of school climate research. Review of Educational Research, 83(3), 357-385.

[5] Zins, J. E., Weissberg, R. P., Wang, M. C., \& Walberg, H. J. (Eds.). (2004). Building academic success on social and emotional learning: What does the research say? New York, NY.

[6] *** CASEL Guide (2015). Effective Social and Emotional Learning Programs. Middle and High School edition. Chicago. [Online]. Available: http://secondaryguide.casel.org/caselsecondary-guide.pdf

[7] *** I-Yes project. [Online]. Available: http://www.iyes-project.eu/index.php/ro/. 